\title{
Infrared imaging spectroscopic system based on a PGP spectrograph and a monochrome infrared camera
}

\author{
P. Beatriz Garcia-Allende*, Francisco Anabitarte, Olga M. Conde, Francisco J. Madruga, Mauro \\ Lomer, Jose M. Lopez-Higuera \\ Photonics Engineering Group, Universidad de Cantabria, Avda. Los Castros s/n, 39005 \\ Santander, Spain
}

\begin{abstract}
Hyperspectral imaging spectroscopy has been widely used in remote sensing. However, its potential for applications in industrial and biological fields is enormous. Observation line spectrographs, based on the reflectance of the material under study in each field, can be obtained by means of an imaging spectrometer. In this way, imaging spectroscopy allows the simultaneous determination of the optical spectrum components and the spatial location of an object in a surface. A simple, small and low-cost spectrometer, such as those ones based on passive Prism-Grating-Prism (PGP) devices, is required for the abovementioned application fields. In this paper a non-intrusive and non-contact near infrared acquisition system based on a PGP spectrometer is presented. An extension to the whole near infrared range of the spectrum of a previously designed system in the Vis-NIR range has been performed. The reason under this investigation is to improve material characterization. To our knowledge, no imaging spectroscopic system based on a PGP device working in this range has been previously reported. The components of the system, its assembling, alignment and calibration procedures will be described in detail. This system can be generalized for a wide variety of applications employing a specific and adequate data processing.
\end{abstract}

Keywords: hyperspectral system, infrared imaging spectroscopy, imaging spectrograph, Prism-Grating-Prism device.

\section{INTRODUCTION}

Imaging spectroscopy has been used in a wide variety of monitoring scenarios, being aerospace remote sensing the most widely known. However, the benefits it can provide in industrial and biological fields, where real-time inspection is desired, are remarkable. Examples of the latter can be found, for example, in the processing of food [1] and tobacco [23]. High accuracy, reliability and real-time operation are required in this kind of application. Therefore, potential application fields are subject to the development of efficient spectral interpretation techniques but also to the investigation in novel image acquisition systems. These systems must be able to collect signature characteristics of the material under analysis to make easier the discrimination task.

A raw quality monitoring system utilizing the imaging spectroscopy technique was developed in the Vis-NIR range [2]. Samples belonged to the tobacco industry where tobacco leaves blends need to be distinguish from a wide variety of materials, such as cardboards, paper of sweets, etc., that are usually intermingled with the leaves due to the manual harvest procedure. Imaging spectroscopy is particularly suitable in this case since it allows the simultaneous determination of the spectrum of the diffused reflected radiation of the material along all the points of an image line, while the mixed materials are transported on the conveyor belt in the production plant. Afterwards, an intelligent interpretation of the images supplies material discrimination. To simultaneously acquire the information of both domains, spectral and spatial, the main component of the system is the equipment known as ImSpector, commercialised by Specim Ltd. It is an imaging spectrograph based on a passive Prism-Grating-Prism (PGP) device [4] because the latter provides the required small size of the collection system. As the discrimination analysis was performed in the Vis-NIR range, the V10E version of Imspector, whose spectral range is from 400 to $1000 \mathrm{~nm}$ was employed.

McClure studies on tobacco leaves' spectrum demonstrate that they have important absorption peaks in 1450 and 1930 $\mathrm{nm}$ [5]. Hence, it is natural to think that the performance of the system can take advantage of the extension of the spectral range over the whole NIR of the spectrum since the effectiveness of the system depends on the presence of the mentioned signature characteristics. This extension requires the replacement of the previously employed PGP device,

Infrared Imaging Systems: Design, Analysis, Modeling, and Testing XIX, edited by Gerald C. Holst, Proc. of SPIE Vol. 6941 ,

694118, (2008) - 0277-786X/08/\$18 - doi: 10.1117/12.770290

Proc. of SPIE Vol. 6941 694118-1 
and a new acquisition system has to be designed and developed because, to our knowledge, no imaging spectroscopic sensors based on this kind of devices and working in this range has been previously reported.

The paper is divided in five sections as follows: in Section 2, the optical technique in which the system is based, imaging spectroscopy, is introduced. Section 3 discusses the main specifications of the element of the acquisition system, while in Section 4 the required calibration procedures of the latter are thoroughly described. Sections 5 and 6 discuss the image collection results and extract several conclusions, respectively.

\section{IMAGING SPECTROSCOPY BACKGROUND}

Spectroscopy is an optical technique which measures the radiant intensity and energy of the interaction between light and any material. In the particular case of absorption spectroscopy the compound interacting with the light selectively absorbs photons depending on their wavelength. The photons that have not been absorbed are either transmitted or reflected by the sample object. In this way, the spectrum of the diffuse reflectance of the material can be obtained. It is the so called "spectral signature" or "spectral fingerprint", which can be used to unambiguously identify the material. The concept of imaging spectroscopy, which is shown in Figure 1, means that a spectrum is measured for each spatial element in the line of vision. Therefore, the captured data will be a three-dimensional data set, defined by spatial $x$ and $y$ and wavelength $\lambda$ coordinates. The system that provides this kind of datum is called hyperspectral imaging spectrometer.

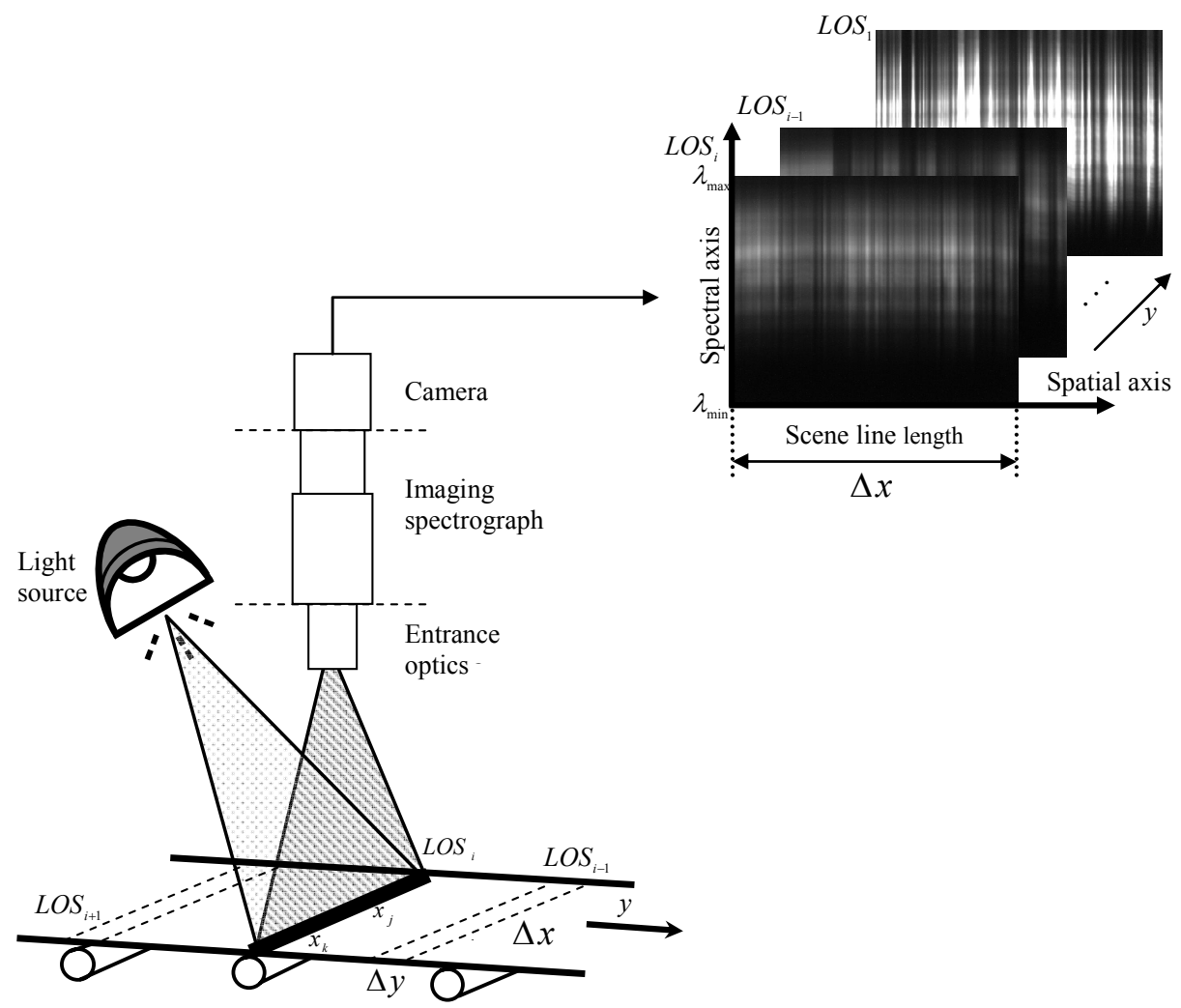

Fig. 1. The concept of imaging spectroscopy and comprehensive block diagram of the system

Figure 1 also depicts the components of an imaging spectroscopy system. The light source, e.g. a halogen lamp with a quasi-flat spectrum, illuminates the object to be measured. Entrance optics collects the radiation from the object and forms an image on the entrance slit of the imaging spectrograph. With application to raw material quality control in industrial scenarios, and by the employment of the appropriate entrance optics, the length of the observation line, or scene line, is fitted to cover the width of the conveyor belt that transports the mixed materials in the production plant. The length $(\Delta x)$ and width $(\Delta y)$ of the instantaneous field of view (LOS, line of sight) depends on the size of the entrance slit of the spectrometer but also on the lens focal length and on the distance between the sample and the lens. The 
radiation from the slit is collimated by either a lens or a mirror and then dispersed by a dispersing element, so that the direction of propagation of the radiation depends on its wavelength. This dispersing element can be a PGP device [5] because it provides the small sizes of the acquisition systems required in application scenarios different from aerospace remote sensing. The dispersed radiation is then focused by the focusing optics, i.e. a lens or mirror. Every point $x_{i}$ is represented by a series of monochromatic images forming a continuous spectrum in the direction of the spectral axis. The focused radiation is detected by the 2D detector array of the camera attached to the spectrometer. The second spatial dimension of the object, $y$, is generated by scanning or moving the field of view of the instrument relative to the scene. In this particular application case is the movement of the conveyor belt which forms this spatial dimension.

This kind of imaging spectroscopy system working in the Vis-NIR range of the spectrum has been employed in multiple monitoring scenarios $[1,6-7]$, due to existence of low-cost commercially available cameras. The effectiveness of such a spectroscopic technique depends on the presence of signature characteristics of the material and therefore it would be advisable to extend the spectral range of analysis. In the particular application case of [2-3] there are also important spectral features in higher wavelengths such as strong water absorption bands in 1450 and $1930 \mathrm{~nm}$ [5] which make worthy the development of an imaging spectroscopy system in the whole NIR range.

\section{NIR IMAGING SPECTROSCOPY SYSTEM}

As mentioned in Section 2, the main component of an imaging spectroscopy system is the imaging spectrograph, because it allows the simultaneous acquisition of the spectral and spatial information. It is attached between an objective and a camera. In this Section the elements of the system and their main specifications are stated. Afterwards assembling and calibration will be described.

The equipment known as Imspector, commercialised by Specim Ltd., has been used as the imaging spectrograph. Its dispersing element is a volume type holographic transmission grating. This grating is used in a prism-grating-prism structure (PGP - element), which provides high diffraction efficiency and good spectral linearity [4]. The version employed in [2-3], which was the V10E with a spectral range from $400 \mathrm{~nm}$ to $1000 \mathrm{~nm}$, has been replaced by the version N24E. The spectral range of the latter is from $1000 \mathrm{~nm}$ to $2400 \mathrm{~nm}$ and its spectral resolution is $8 \mathrm{~nm}$.

A monochrome camera is required to record the images provided by the spectrograph. Due the working operation spectral range of the version N24E of the spectrograph, it is not possible to choose among a wide variety of possibilities because sensitive detectors in such spectral ranges are expensive. The camera XEVA-FPA-2.5-320 by Xenics is used. It is based in a $\mathrm{HgCdTe}$ array detector with 320 x 256 pixels and a total dynamic range of 14 bits. The shorter axis of the sensor is the spectral one and its range is from 850 to $2500 \mathrm{~nm}$. The camera is connected to a computer for control and acquisition, being Camera Link the interface for image transference and USB for camera control. The manufacturer provides a privative software called $X$-Control to perform the control of camera parameters such as integration time or cooler power and image parameters (contrast, brightness, etc.). It is not possible, however, to perform automatically the alignment of the two assembled components, camera and spectrograph, and this is a key point in the effectiveness of the acquisition system because every pixel of the spectral axis must correspond with the same wavelength along all the spatial points of the line of vision.

A Pentax C31634 has been the employed lens. Its focusing range and numerical aperture are manually controlled.

\section{ALIGNMENT AND CALIBRATION}

Alignment and calibration procedures are key points in the arrangement of a hyperspectral imaging system. Light sources with multiple and known emission lines are required to perform this task. As no applications have been found in the NIR range, the aim of this article is to provide a detailed calibration procedure when a multiple-line light source is not at one's disposal. 


\subsection{Axis alignment}

The spatial axis of the spectrograph has to be parallel to horizontal pixel lines of the camera. In the Vis-NIR range a laser of $670 \mathrm{~nm}$ and an $\mathrm{Hg}$-Ar lamp where employed to performed this task. The hyperspectral image of the latter is shown in Figure 2.

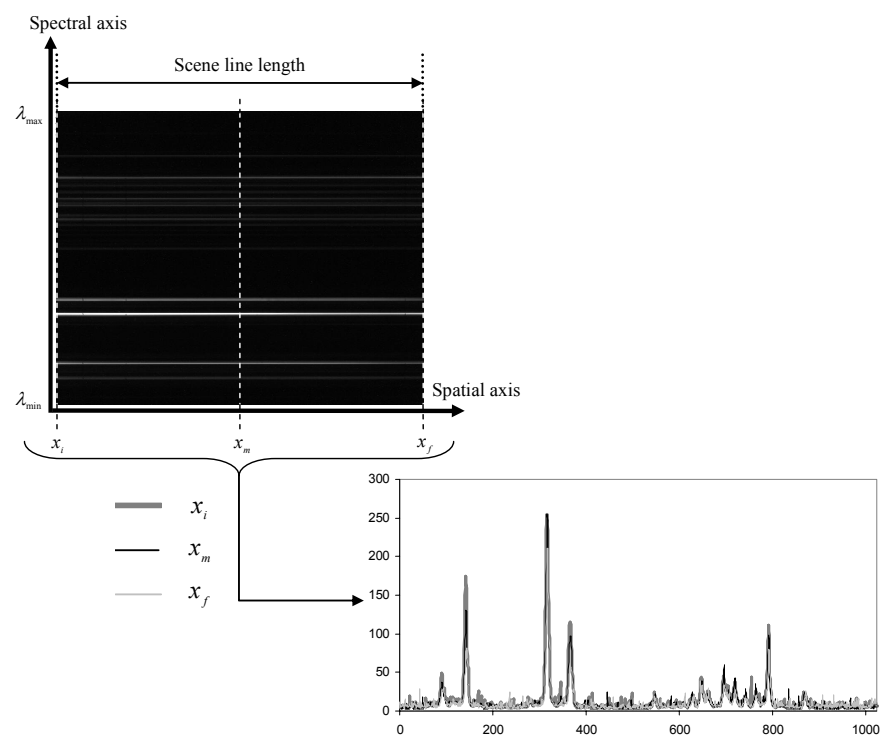

Fig. 2. Hyperspectral image of the Hg-Ar multi-line source.

To deal with the unavailability of such a multi-line light source in the operating spectral range of the system, the set-up depicted in Figure 3 was considered. A tunable laser source whose output wavelength was fixed to $1680 \mathrm{~nm}$, and two laser sources of $1550 \mathrm{~nm}$ and $1310 \mathrm{~nm}$, respectively, were employed. The aim was to cover the whole entrance slit of the imaging spectrograph. In this way, the same wavelength is collected in different spatial pixels and axis alignment can be performed. Although the six FC/PC connectors where placed as much close as possible, the set-up did not succeed. The reason is that, as axis alignment has to be done with no focusing optics, it is impossible to have an enough length of line of vision.

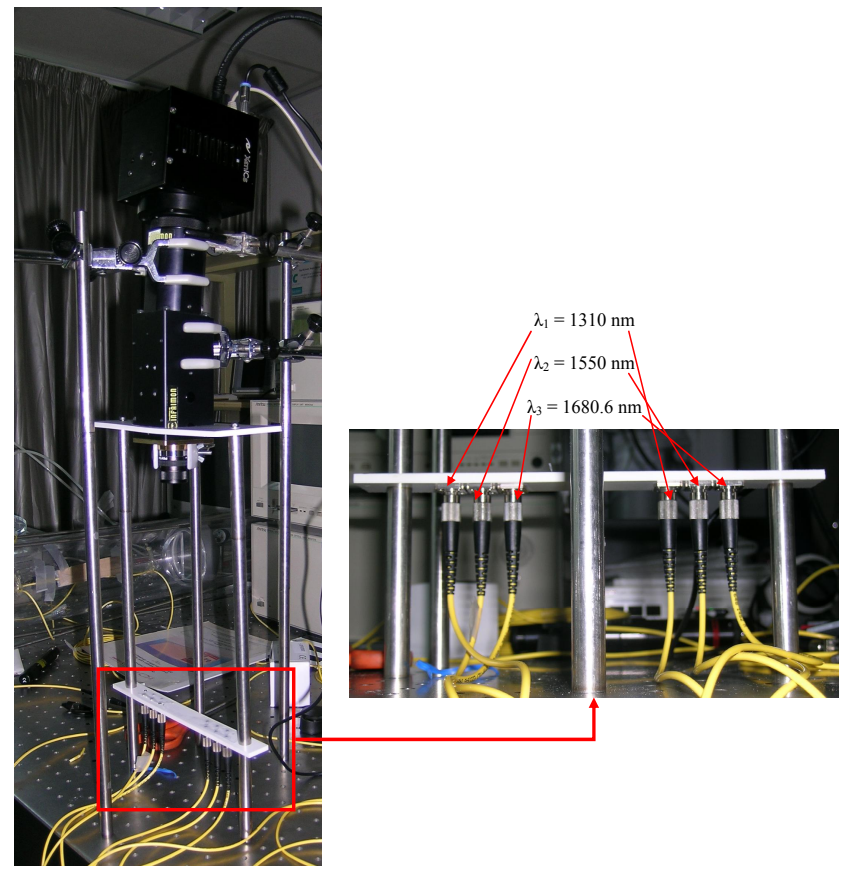

Fig. 3. Calibration attempt based on independent spatial points per wavelength. 
To entirely cover the entrance slit, another alternative was implemented. Its block - diagram is depicted in Figure 4 . The same light sources as previously were employed, but now by means of two fiber optic couplers and a manual positioning system, the three wavelengths are concentrated in a spatial point, the tip of the optical fiber. The numerical aperture of the fiber allows the filling of the whole entrance slit of the imaging spectrograph. The inset of Figure 4 shows how the end of the fiber is placed in front of the spectrograph slip.

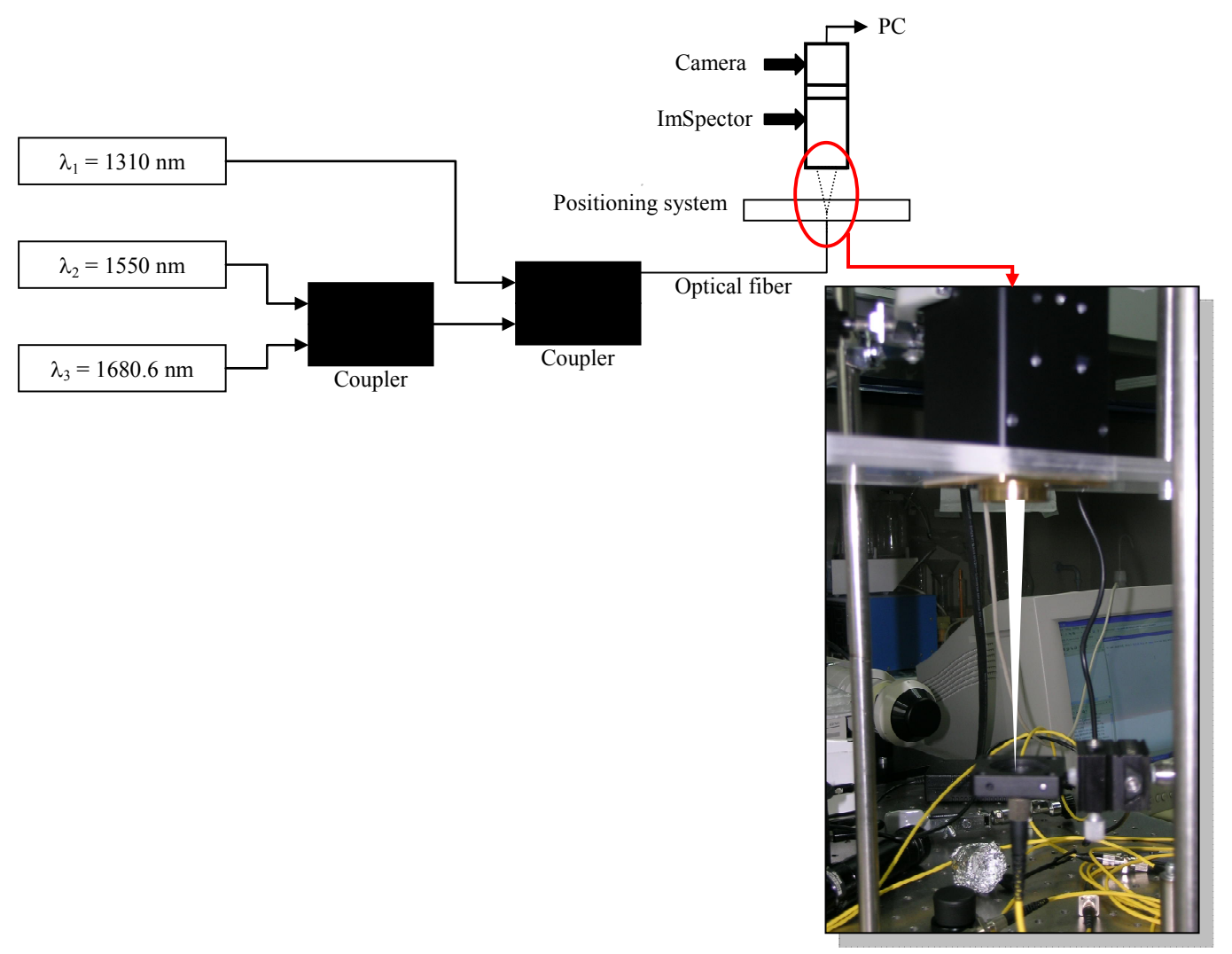

Fig. 4. Final calibration set-up with spatial superposition of different wavelengths.

Once the set-up has been arranged, axis alignment is performed by turning the camera with respect to the spectrograph. The effect of this calibration can be seen in Figure 5, where the spatial axis is placed horizontally and the spatial axis is vertical. Therefore, considering this axis definition, the system will be calibrated when the spectral lines are at the same height at the left and right side of the detector. A software program was developed with Matlab® 7.0 (R14) [8] to process the collected image. It performs a peak searching to assure that the maximum is in the same pixel number along the whole line of vision.

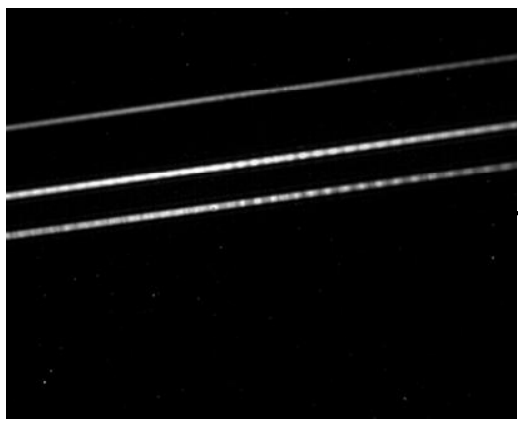

a)

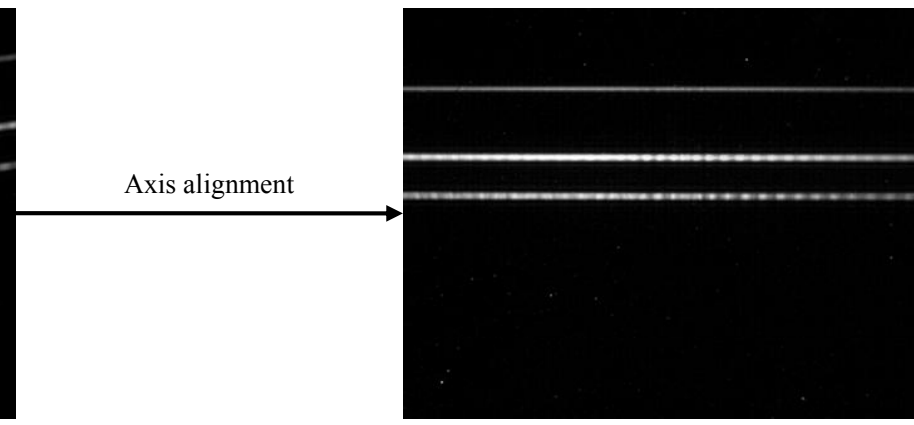

b)

Fig. 5. Effect of axis alignment calibration procedure: a) hyperspectral image collected by the system before calibration; b) imaged captured after performing axis alignment. 


\subsection{Spectral axis calibration}

The wavelength calibration process consists in defining the spectral axis. In the approach followed here, the relationship between the spectral wavelength and the pixel number of the corresponding spectral axis of the detector is expressed by means of:

$$
\lambda(p)=C_{0}+C_{1} p+C_{2} p^{2}
$$

where $\lambda$ is the wavelength associated to pixel $p, C_{0}$ is the wavelength corresponding to pixel 0 or intercept, $C_{1}$ and $C_{2}$ are constants to be determined. The set-up and peak searching software developed to perform axis alignment were employed again. The latter provides an estimation of the central wavelength of those emission lines and then a linear regression can be performed and, therefore, $C_{0}, C_{1}$ and $C_{2}$ can be obtained.

Given equation (1), and considering the three pairs of known emission line central wavelength - CCD pixel number the unknown coefficients can be determined by trying to minimize the following expression:

$$
S=\sum_{i=0}^{2}\left[\lambda\left(p_{i}\right)-y_{i}\right]^{2}
$$

where $y_{i}$ represents the known wavelengths. This method is commonly referred to as linear least squares regression.

Considering the partial derivatives of $S$ with respect to the coefficients, and equalling to zero, the resulting equation system for an $2^{\text {nd }}$-order polynomial would be given by:

$$
\left(\begin{array}{ccc}
3 & \sum_{i=0}^{2} p_{i} & \sum_{i=0}^{2} p_{i}^{2} \\
\sum_{i=0}^{2} p_{i} & \sum_{i=0}^{2} p_{i}{ }^{2} & \sum_{i=0}^{2} p_{i}{ }^{3} \\
\sum_{i=0}^{2} p_{i}{ }^{2} & \sum_{i=0}^{2} p_{i}{ }^{3} & \sum_{i=0}^{2} p_{i}{ }^{4}
\end{array}\right)\left(\begin{array}{c}
C_{0} \\
C_{1} \\
C_{2}
\end{array}\right)=\left(\begin{array}{c}
\sum_{i=0}^{2} y_{i} \\
\sum_{i=0}^{2} y_{i} p_{i} \\
\sum_{i=0}^{2} y_{i} p_{i}{ }^{2}
\end{array}\right)
$$

This process allows to establish the following calibration polynomial:

$$
\lambda(p)=975.2213+6.554 p-0.0045 p^{2}
$$

Given that the shortest axis of the camera is the spectral one, the spectral range of the whole system goes from 975.2213 $\mathrm{nm}$ to $2358.1333 \mathrm{~nm}$.

The spectral resolution of the system depends on the width of the entrance slit of the spectrograph but also on the camera resolution. As mentioned in Section 3, the version N24E of the commercial equipment known as Imspector, whose spectral resolution is $8 \mathrm{~nm}$, is used. Secondly, considering the obtained spectral range and that the camera resolution is 320 (spatial axis) x 256 (spectral axis), system resolution would be:

$$
\Delta \lambda=\frac{\lambda_{\max }-\lambda_{\min }}{\text { wavelength number }}=\frac{2358.1333-975.2213}{256} \approx 5.4 \mathrm{~nm}
$$

which is smaller. System resolution is, therefore, $8 \mathrm{~nm}$ and it is restricted by the imaging spectrograph.

\subsection{Spatial calibration}

The aim of this calibration procedure is to determine the dimensions, length and width, of the line of vision. They depend on the slit length and width, but also on the distance between target and objective lens and on lens focal length. The latter has to be adjusted to have the target sample in focus, and, therefore, the field of view has to be experimentally determined. A fringe pattern, as the one depicted in Figure 6, was displaced under the system in order to perform this calibration procedure. The inset of Figure 6 depicts the hyperspectral image collected when the pattern suits the field of view of the system. The definition of the lines shows how the focus is correctly adjusted. A field of view of $143 \times 15 \mathrm{~mm}$ is obtained, with a working distance of approximately $26 \mathrm{~cm}$. 


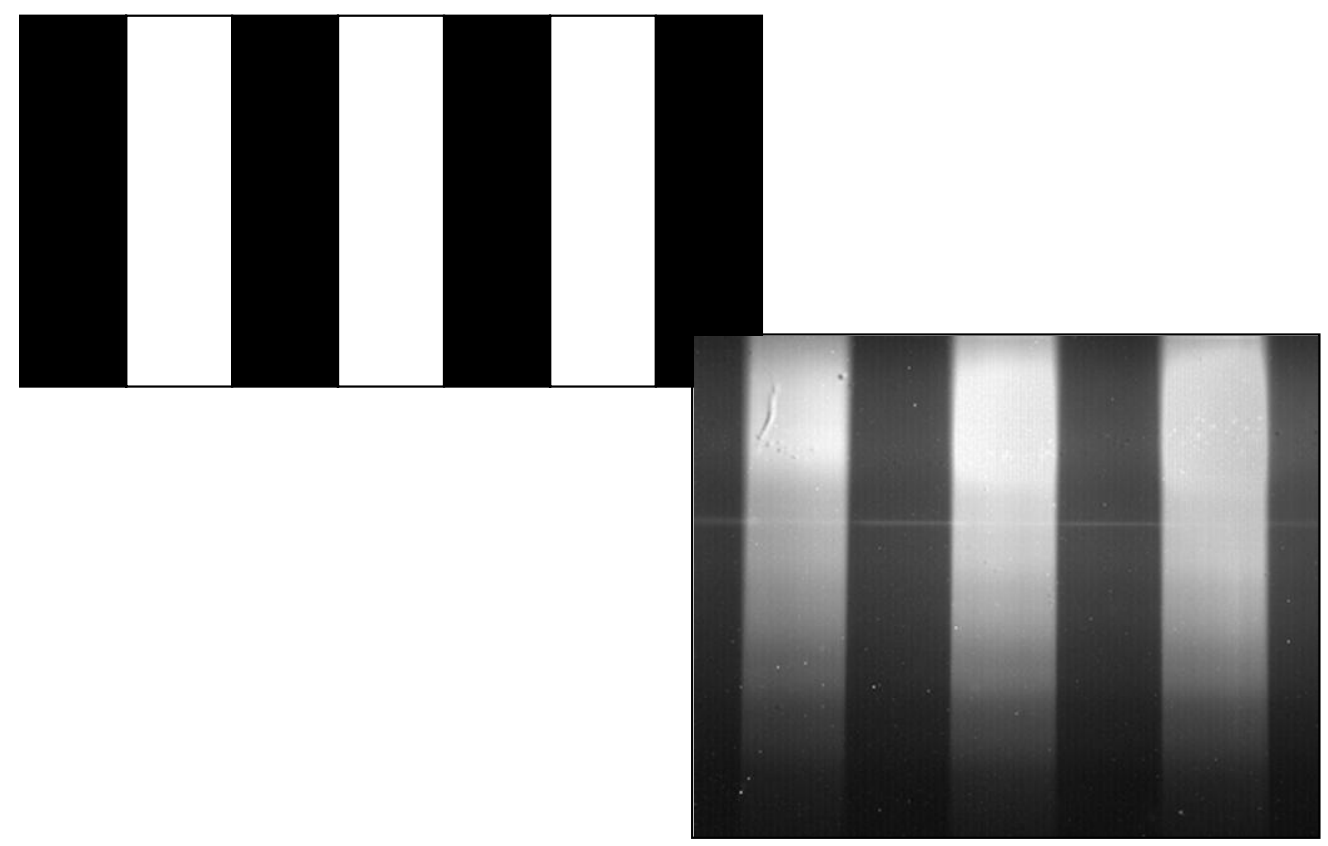

Fig. 6. Fringe pattern employed in spatial calibration procedure and its corresponding hyperspectral image.

\subsection{Responsivity calibration}

Due to the non-linear spectral response of the camera, the flat standard reflectance measurement does not result in a flat curve. Therefore, a reflectance calibration, in the working spectral range, is required. To perform this step, a dark image and an image of white reference are captured. The dark image is obtained by blocking the light entering the lens, while the white reference is the reflectance measurement of a standard material called Spectralon. Once the sample image has been acquired, the sample reflectance at all wavelengths $(\lambda)$ and spatial pixels of the observation line $(x)$ is calculated as follows:

$$
R\left(\lambda_{i}, x_{i}\right)=\frac{S\left(\lambda_{i}, x_{i}\right)-D\left(\lambda_{i}, x_{i}\right)}{W\left(\lambda_{i}, x_{i}\right)-D\left(\lambda_{i}, x_{i}\right)}
$$

where $S\left(\lambda_{i}, x_{i}\right)$ is the sample value at wavelength $\lambda_{i}$ and spatial position $x_{i}, D\left(\lambda_{i}, x_{i}\right)$ is the value for the dark reference and $W\left(\lambda_{i}, x_{i}\right)$ is the equivalent for the white reference.

This calibration compensates for offsets due to the CCD dark current, the light source colour temperature drift, and the lighting spatial non-uniformity across the scene line. Therefore, the sample reflectance is separated from the system response. The effects of reflectance calibration on a hyperspectral image are shown in Figure 7. 

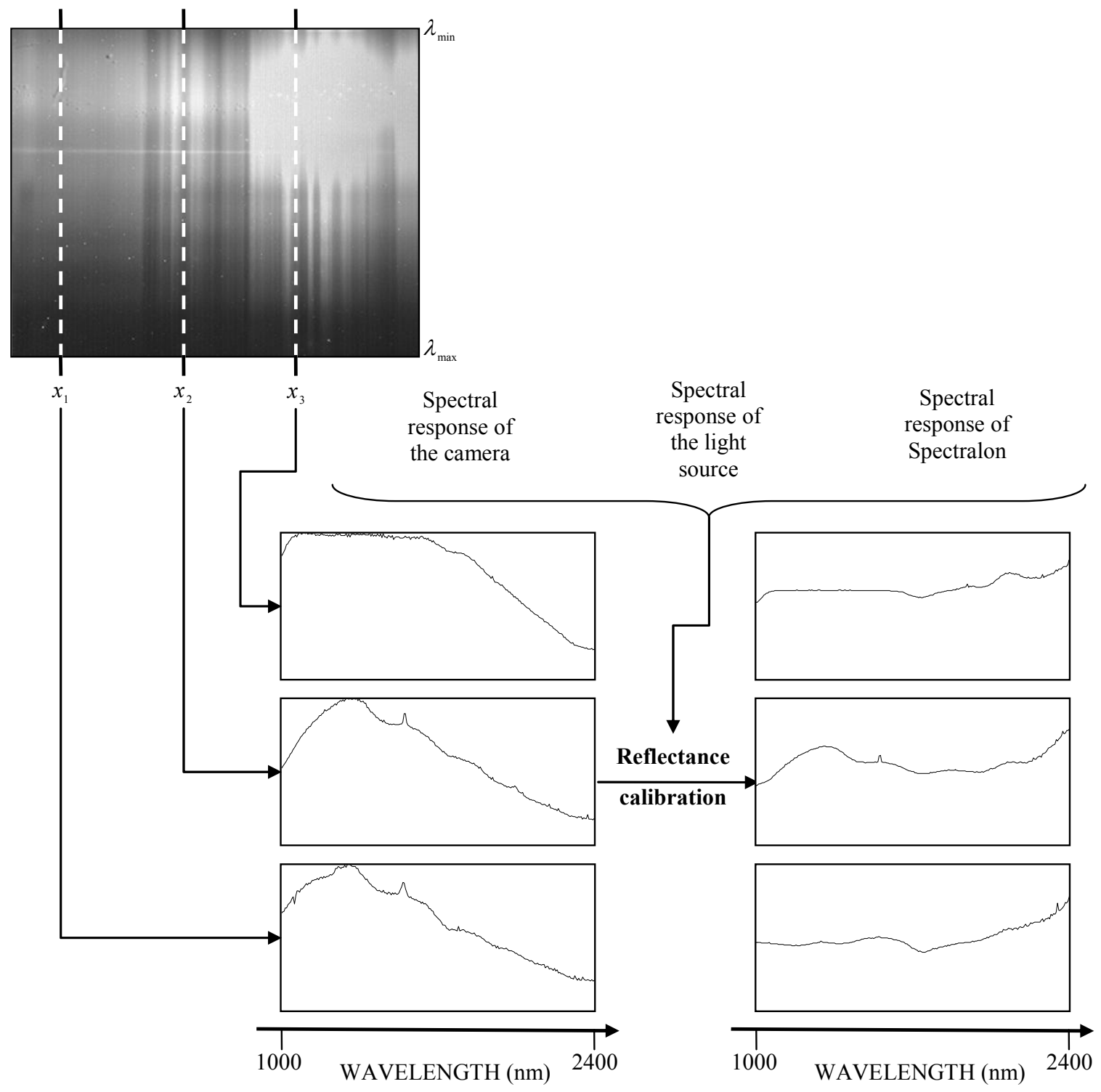

Fig. 7. Reflectance calibration scheme

\section{MATERIAL CHARACTERIZATION}

In this Section the simulation of a scene scenario is performed. The application case is the same that the one developed in [2-3], where the samples under analysis belonged to two different classes with application to raw material quality control. The first class is composed by samples consisting of tobacco leaves. And the second class is composed by undesired materials that typically appear in tobacco industry production plants due to the manual harvest procedure of the leaves. This class includes wood, cardboard, different colored cellophanes, leather, foil, paper of sweets, textile threads and brown and green leaves of other vegetable materials different from tobacco. This scenario simulation is summarized in Figure 8, where different regions corresponding in the different materials can be observed. This differentiation is obtained by the adequate performance of the abovementioned calibration procedures.

A real photograph of the sample under analysis, where the white lines delimit the line of vision, is depicted in Figure 8 a), while Figure $8 \mathrm{~b}$ ) presents a zoom of the line of vision captured by the spectrograph. In this sample four different 
regions can be observed corresponding to cardboard, tobacco leaves, paper of sweet and tobacco leaves, respectively. Figure $8 \mathrm{c}$ ) depicts the associated hyperspectral image associated, in which the four different areas mentioned above can be clearly distinguished. Therefore, the efficiency of the developed hyperspectral system to capture the features of each material under analysis has been successfully proved.

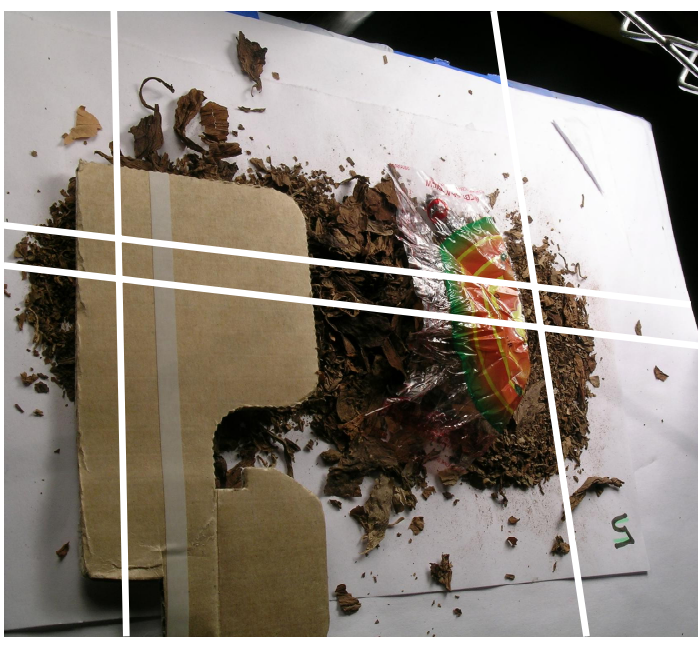

a)

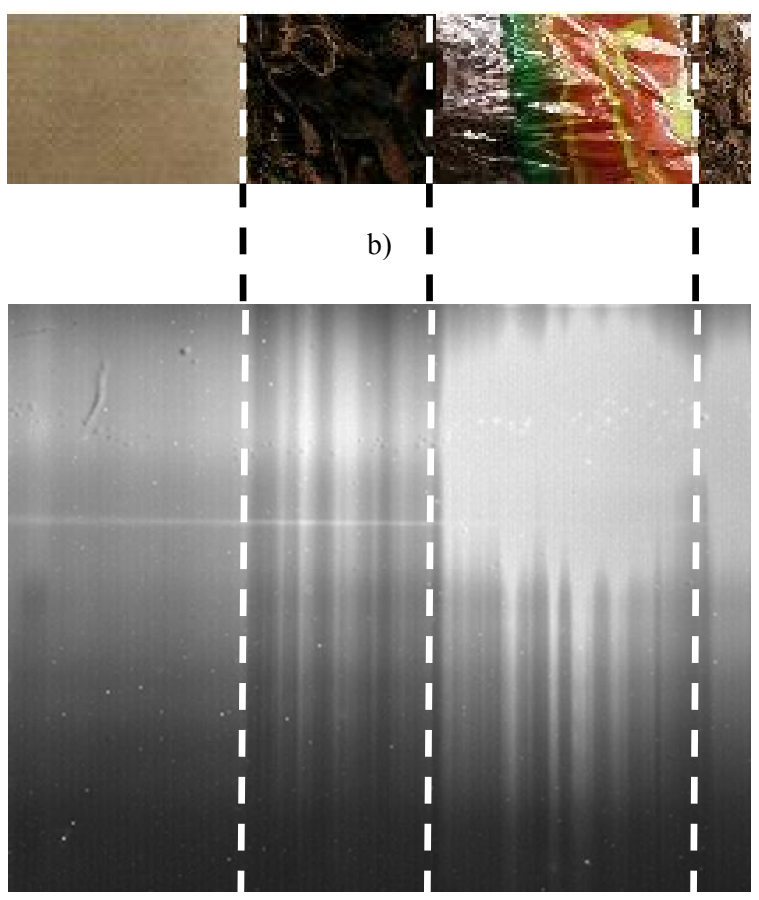

c)

Fig. 8. Environment simulation. a) Sample under analysis; b) Zoom of the scene line; c) Corresponding hyperspectral image

\section{CONCLUSIONS AND FUTURE WORK}

A hyperspectral system in the NIR range has been designed and developed. In addition, it has been successfully validated with application to material discrimination. Since no imaging system working in this region of the spectrum has been previously reported, its components have been described. Special attention has been paid to calibration problems and procedures. The commercial availability of calibration lamps working in these wavelengths is very recent. Therefore, calibration procedures when such a calibration lamp it is not at one's disposal have been here presented.

Further work is being pursued with respect to the optimization of the illumination and the post-processing software of the captured images. The halogen foodlights used at present are wanted to be substituted by an array of LEDs with their peak wavelengths matching those of the features of the material under analysis. The aim is to reduce integration times to allow the operation of the system in real time in practical application. The latter is also the target of the different interpretation algorithms of the images that are under evaluation.

\section{ACKNOWLEDGEMENTS}

This work has been co-supported by the Science and Technology Ministry of the Spanish Government through the TEC'2005-08218-C02-02 and TEC'2007-67987-C02-01 projects. The authors also thank Infaimon Company and its staff, especially X. Aramendi and C. López and C. Carreté, for their valuable help during the arrangement of the hyperspectral imaging system. 


\section{REFERENCES}

1 G. ElMasry, N. Wang, A. ElSayed and M. Ngadi, "Hyperspectral imaging for nondestructive determination of some quality attributes for strawberry”, J. Food Eng. 81, 98-107 (2007).

2 P.B. Garcia-Allende, O.M. Conde, A.M. Cubillas, C. Jauregui, J.M. Lopez-Higuera, "New raw material discrimination system based on a spatial optical spectroscopy technique", Sens. Actuator A-Phys. 135, 605-612 (2007).

3 P.B. Garcia-Allende, O.M. Conde, J. Mirapeix, A. Cobo and J.M. Lopez-Higuera, "Quality control of industrial processes by combining a hyperspectral sensor and Fisher's linear discriminant analysis”, Sens. Actuator B-Chem. 129, 977-984 (2008).

4 T.Vaarala, M. Aikio, H.Keraenen, "An advanced prism-grating-prism imaging spectrograph in on-line industrial applications", Proc. SPIE 3101 (1997) 322-330.

5 W.F. McClure, "Spectral characteristics of tobacco in the near-infrared region from 0.6 to 2.6 microns", Tobacco Sci. 12 (1968) 232-235.

6 J. Xing, C. Bravo, T. Pál, H. Jancsók, J.R. Baerdemaeker, “Detecting bruises on 'Golden Delicious' apples using hyperspectral imaging with multiple wavebands", Biosys. Eng. 90 (2005) 27-36.

B. Andre Weinstock, J. Janni, L. Hagen, S. Wright "Prediction of oil and oleic acid concentrations in individual corn (Zea mays L.) kernels using near-infrared reflectance hyperspectral imaging and multivariate analysis," Appl. Spectrosc. Vol. 60, pp. 9-16, 2006.

$8 \quad$ Matlab® Reference Manual, The Mathworks Inc., MA, USA. 